Responsabile: Leonardo Morlino - Stampa: Grafiche Galeati - Imola, con i tipi del Centro Immagine Snc - Capannori (LU). Registrato al n. 4110 presso la Cancelleria del Tribunale Civile Penale di Bologna, il 14-12-1970 - Copyright (C) 1997 by Società editrice il Mulino, Bologna. 


\section{RIVISTA}

ITALIANA

DI

SCIENZA

POLITICA

\section{N. $1 / 1994$}

Razionalità politica, virtù civica e identità nazionale, di Gian Enrico Rusconi. - Legittimità, consolidamento e crisi nell'Europa meridionale, di Leonardo Morlino e José Ramon Montero. - Le tre ondate dell'estremismo di destra in Germania occidentale, di Ekkart Zimmermann e Thomas Saalfeld. - Elezioni locali e destrutturazione partitica. La nuova legge alla prova, di Aldo Di Virgilio.

\section{N. 2/1994}

Nuovi conflitti e nuovi sistemi partitici all'est. Verso un modello esplicativo, di Pietro Grilli di Cortona. - La nazionalizzazione del voto, di Daniele Caramani. - La nuova legge elettorale in Parlamento: chi, come e perché, di Adriano Pappalardo. - Il voto di approvazione: il doppio turno in un turno solo, di Roberto D'Alimonte. - Partiti di centro e partiti centrali: una chiarificazione concettuale, di Reuven Y. Hazan.

\section{N. $3 / 1994$}

Una vigilia di incertezze, di Giacomo Sani. - Media, sondaggi e spese elettorali: la nuova disciplina, di Carlo Fusaro. - I programmi elettorali e il ruolo dei mass media, di Paolo Segatti. - Dai partiti ai poli: la politica delle alleanze, di Aldo Di Virgilio. - I candidati, di Liborio Mattina. - Il voto proporzionale: il nuovo spazio politico italiano, di Luca Ricolfi. - La competizione maggioritaria: le origini elettorali del Parlamento diviso, di Stefano Bartolini e Roberto D'Alimonte. - Gli effetti distorsivi del nuovo sistema elettorale, di Alessandro Chiaramonte. - Gli eletti, di Luca Verzichelli.

\section{N. $1 / 1995$}

Elogio del semi-presidenzialismo, di Giovanni Sartori. - Plurinazionalismo e democrazia, di Juan J. Linz. - Teoria della scelta razionale e scienza politica: un incontro con pochi frutti?, di Donald $P$. Green e lan Shapiro. - I nuovi sindaci: come cambia una carriera politica, di Gianfranco Bettin Lattes e Annick Magnier. - L'alta burocrazia ministeriale: modelli di reclutamento e carriera, di Rosalba Chiarini. 


\section{N. $2 / 1995$}

Democrazia e referendum, di Yannis Papadopoulos. - La politicizzazione di un potere neutrale. Magistratura e crisi italiana, di Stella Righettini. - La strutturazione dei sistemi partitici nelle nuove democrazie, di Maurizio Cotta. - Modelli teorici di federalismo, di Daniela Giannetti. Apprendimento e cambiamento nelle politiche pubbliche. II ruolo delle idee e della conoscenza, di Elisabetta Gualmini.

\section{N. $3 / 1995$}

La crescita dei poteri regolativi nella comunità europea, di Giandomenico Majone. - La presidenza della commissione europea. Vincoli e risorse, di Ken Endo. - L'elettore che ragiona. Il caso dei sistemi multipartitici, di Franz Urban Pappi. - La transizione italiana: il voto regionale del 23 aprile, di Roberto D'Alimonte.

\section{N. $1 / 1996$}

Riflessioni sulla metodologia degli studi comparati, di Neil J. Smelser. - Integrazione europea e welfare states nazionali, di Fritz $W$. Scharpf. - II modello sud-europeo di welfare state, di Maurizio Ferrera. - Dal pluralismo polarizzato al pluralismo moderato. II modello di Sartori e la transizione italiana, di Adriano Pappalardo. - Federalismo e movimenti sociali, di Marco G. Giugni.

\section{N. 2/1996}

Cosa è «competizione» in politica e come va studiata, di Stefano Bartolini. - L'espansione del potere giudiziario nelle democrazie contemporanee, di Carlo Guarnieri e Patrizia Pederzoli. La formazione dei partiti confessionali in Europa, di Stathis $N$. Kalyvas. - Alla ricerca della collegialità di governo: i vertici di maggioranza dal 1970 al 1994, di Annarita Criscitiello. - I gruppi parlamentari dopo il 1994. Fluidità e riaggregazioni, di Luca Verzichelli.

\section{N. $3 / 1996$}

Elezioni politiche 1996, a cura di Roberto D'Alimonte e Stefano Bartolini. - Programmi, media e opinione pubblica, di Giacomo Sani e Paolo Segatti. - I candidati. Visioni politiche e carriere, di Liborio Mattina e AlessandroTonarelli. - Le alleanze elettorali. Identità partitiche e logiche coalizionali, di Aldo Di Virgilio. - La partecipazione elettorale: gli effetti della competizione maggioritaria, di Daniele Caramani. - Indizi di un inverno precoce: il voto proporzionale tra equilibrio e continuità, di Roberto Cartocci. - Come perdere una maggioranza. La competizione nei collegi uninominali, di Roberto D'Alimonte e Stefano Bartolini. - L'effetto mancato della riforma maggioritaria: il voto strategico, di Alessandro Chiaramonte. - La classe politica della transizione, di Luca Verzichelli. • 


\section{Novità gennaio-aprile 1997}

Pier Paolo Giglioli, Sandra Cavicchioli

$$
\text { e Giolo Fele }
$$

Rituali di degradazione

Anatomia del processo Cusani saggi pp. 244 , L. 28.000

Come un'intera classe politica è stata simbolicamente spogliata di un potere che sembrava inespugnabile

\section{Carlo Baccetti II Pds}

Verso un nuovo modello di partito? studi e ricerche, pp. 286, L. 30.000

Un quadro completo e dettagliato delle trasformazioni in atto nella maggiore formazione politica della sinistra italiana
Bino Olivi

e Bruno Somalvico

La fine della comunicazione di massa

Dal villaggio globale

alla Babele elettronica

studi e ricerche, pp. 392 , L. 45.000

Una nuova era comunicativa si apre con il superamento della televisione generalista e l'awento della pay-tv

\section{Marco Tarchi}

Dal Msi ad An

Organizzazione e strategie studi e ricerche, pp. 420 , L. 35.000

Un'analisi approfondita del partito "postfascista" e dei suoi rapporti con la società civile durante una complessa transizione
Nicola Rossi

Per un pezzo

di carta

Stato e prospettive dell'istruzione in Italia

studi e ricerche, pp. 400 , L. 45.000

Una valutazione dello stato dell'istruzione in Italia: ragioni dell'arretratezza e modalità di rilancio

Roberto D'Alimonte e Stefano Bartolini Maggioritario per caso Le elezioni politiche del 1996 studi e ricerche, pp. 376, L. 40.000

II sistema uninominale alla sua seconda prova: analisi, interrogativi,

e opportunità di riforme
Rodolfo Lewanski Governare l'ambiente Attori e processi della politica ambientale studi e ricerche, pp. 296, L. 35.000

Uno strumento per analizzare e migliorare la prestazione delle politiche per l'ambiente in termini di efficacia, efficienza ed equità

\author{
Aldo Piperno \\ Mercati assicurativi e istituzioni \\ La previdenza sanitaria integrativa \\ studi e ricerche, pp. 264, L. 32.000 \\ Una proposta di riforma \\ per razionalizzare le risorse esistenti con \\ una diversa divisione delle prestazioni \\ tra sistema sanitario nazionale \\ e assicurazioni private
}

Dal momento che d un lettoro delle riste da Wuino, abbiamo deciso di riservale condialoni d’ecefísto

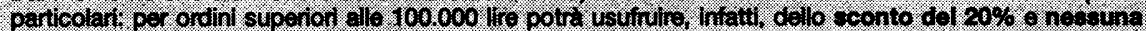

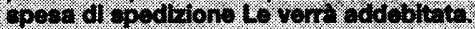

Desidero acquistare i seguenti volumi:

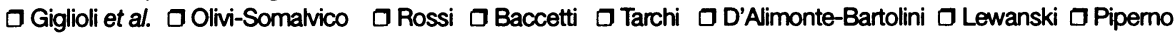
口 Dal momento che il mio acquisto supera L. 100.000 , applicatemi lo sconto del $20 \%$.

Per il pagamento:

addebitate sulla mia carta di credito

$\square$ inviate i volumi contrassegno

D American Express

$\square$ Visa-Mastercard

N. scad. (solo per enti) I

Data .......................... Firma

Nome e cognome/Ragione sociale

Indirizzo

Località

Cap

Prov.

Partita Iva/Codice fiscale

Tel. 


\section{il Mulino}

\section{Novità della collana «il Mulino/Tendenze»}

\section{Tommaso Padoa-Schioppa \\ Il governo dell'economia}

pp. 96, L. 10.000

Premessa. - Agenda e non-agenda. - La politica e il mercato. - La costituzione economica dell'Italia. - Cinquant'anni: progresso e ritardi. - Gli strumenti del govemo dell'economia. - Ulisse e le sirene.

\section{Gianfranco Pasquino La democrazia esigente}

pp. 84, L. 10.000

Premessa. - Le opportunità della democrazia. - L'etica nella politica democratica. L'etica pubblica tra convinzione e responsabilità. - La perfettibilità delle democrazie.

\section{Gian Enrico Rusconi Patria e repubblica}

pp. 84, L. 10.000

Patriottismo e repubblicanesimo. - Che cosa è il repubblicanesimo. - Cittadinanza e nazione. - Morte della patria e nascita della repubblica. - Patriottismo espiativo e patriottismo costituzionale. - Cittadinanza europea e «popolo» europeo.

Già usciti nella stessa collana

Arnaldo Bagnasco

L'Italia in tempi di cambiamento politico

Edmondo Berselli

L'Italia che non muore 


\title{
il Mulino
}

Remo Bodei

Libro della memoria e della speranza

Guido Bolaffi

Una politica per gli immigrati

Filippo Cavazzuti

Privatizzazioni imprenditori e mercati

\author{
Carlo Azeglio Ciampi \\ Un metodo per governare
}

Antonio Gambino

Gli altri e noi: la sfida del multiculturalismo

Gino Giugni

Socialismo: l'eredità difficile

Arrigo Levi

Le due fedi

Indro Montanelli

Una voce poco fa

Angelo Panebianco

II prezzo della libertà

Romano Prodi

II capitalismo ben temperato

Michele Salvati

Sinistra o cara

Giovanni Sartori

Come sbagliare le riforme 


\section{RASSEGNA ITALIANA DI SOCIOLOGIA}

\begin{tabular}{lll}
\hline Anno trentottesimo & Numero 1 & Marzo 1997
\end{tabular}

\section{LA DISEGUAGLIANZA OGGI}

Studiare la diseguaglianza oggi

Giovanna Procacci

Richiesti ma non benvenuti

ARISTIDE R. ZOLBERG

Diseguaglianze e vulnerabilità sociale

ROBERT CASTEL

Differenze culturali, territorio e cittadinanza in Francia

JACQUELINE Costa-LASCouX

La povertà nella Germania riunificata

HANS-Jürgen ANDRESS

Donne e uomini nella formazione del mercato del lavoro: il caso della Russia

Elena Mezentseva

\section{SAGGI E RICERCHE}

Attori, istituzioni e stato nell'economia della Russia post-sovietica: da transizione a trasformazione

Cristiano Codagnone

\section{LESSICO}

Memoria

PAOLO JEDLOWSKI

\section{NOTE CRITICHE}

Circoli virtuosi della cultura e nuovo Mezzogiorno

CARMEN LECCARDI

Un fascicolo Lit. 32.000. Abbonamento annuo Lit. 128.000 (90.000 per i privati)

Società editrice il Mulino - Strada Maggiore, 37 - 40125 Bologna

Tel. fax ufficio commerciale 051/256041 


\section{il Mulino}

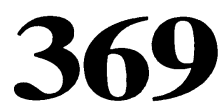

Bologna

anno XLVI

gennaio-febbraio 1997

\section{Sommario}

Ai lettori

Moneta unica, rivoluzione copernicana, di Michele Salvati

Un'America all'italiana, di Sidney Tarrow

Diceria del Cinquantasei. In margine a un anniversario, di Federigo Argentieri

Giovani verso il Duemila

La lunga transizione alla vita adulta, di Alessandro Cavalli

Nuove generazioni: l'Europa è lontana, l'Italia un po' meno, di Ilvo Diamanti

La politica immaginaria, di Luca Ricolfi

\section{Osservatorio scuola}

La paura della storia contemporanea, di Marcello Flores

L'insegnamento della storia nella nostra scuola, di Roberto Vivarelli

Scuola, lavoro e riforma Berlinguer, di Luciano Benadusi

\section{Osservatorio italiano}

Elogio dei ribaltoni, di Gianfranco Pasquino

Misteri di partito, di Paolo Prodi

Caste, non classi. Una società immobile, di Francesca Fabbri e Nicola Rossi

Una variabile fastidiosa. La stagnazione salariale in Italia, di Luigi Campiglio

Il sindacato contro il mercato, di Giuliano Cazzola

I cattolici praticanti e i media, di Franco Garelli

Per non perdere lo stato sociale

Riforma di un welfare diseguale. Limiti e prospettive di cambiamenti possibili, di Chiara Saraceno

Sanità, le insidie all'universalismo, di George France

Pensioni e privatizzazioni: una proposta per la politica economica italiana, di Luca Beltrametti

\section{Cronache}

Un fascicolo Lit. 18.000. Abbonamento annuo Lit. 80.000 Società editrice il Mulino - Strada Maggiore, 37 - 40125 Bologna Tel. fax ufficio commerciale 051/256041 


\section{STATO \\ E MERCATO}

numero 49, aprile 1997

\section{SOMMARIO}

\section{SAGGI E RICERCHE}

Il futuro della diversità dei capitalismi

di Colin Crouch e Wolfgang Streek

Un commento

di Ronald Dore

I processi decisionali nei comuni italiani

di Luigi Bobbio

Non c'è rete senza nodi. Il ruolo del capitale sociale nel mercato del lavoro di Paolo Barbieri

\section{RASSEGNE E DISCUSSIONI}

Sociologia ed economia del mercato del lavoro di Gabriele Ballarino e Fabrizio Bernardi

Ash Amin e Philippe Schmitter discutono su Associative Democracy di Paul Hirst

Un fascicolo Lit. 30.000 . Abbonamento annuo Lit. 90.000 (72.000 per i privati) Società editrice il Mulino - Strada Maggiore, 37 - 40125 Bologna Tel. fax ufficio commerciale 051/256041

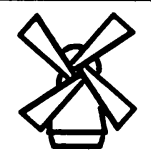


SOMMARIO

\section{SAGGI}

Giovan Francesco Lanzara, Perché è difficile costruire un'istituzione

RICERCHE

Jens Alber, Il ripensamento del welfare state in Germania e negli Stati Uniti Elisabetta Gualmini, L'evoluzione degli assetti concertativi in Italia e in Germania

\section{NOTE}

Chiara De Micheli, L'attività legislativa dei governi al tramonto della Prima Repubblica

\section{RECENSIONI}

